\title{
SOLAR SYSTEM STUDIES WITH THE ARECIBO PLANETARY RADAR SYSTEM
}

\author{
Donald B. Campbell', John K. Harmon ${ }^{2}$ and Michael C. Nolan ${ }^{2}$
}

\author{
${ }^{1}$ National Astronomy and Ionosphere Center, Space Sciences Building, Cornell University, Ithaca, NY 14853, USA \\ (campbell@astro.cornell.edu) \\ ${ }^{2}$ National Astronomy and Ionosphere Center, Arecibo Observatory, Arecibo 00612, Puerto Rico
}

\begin{abstract}
The $305 \mathrm{~m}$ Arecibo telescope equipped with a $1 \mathrm{MW} 2.38 \mathrm{GHz}$ transmitter is used for studies of the terrestrial planets, planetary satellites including the Moon and small bodies in the solar system. Much of the recent program emphasis has been on astrometric and characterization observations of near-Earth objects for which the Arecibo radar system has capabilities not matched by any other Earth-based telescope. However, studies of the surfaces of Mercury, the Moon and Mars continue and renewed observations of Venus are planned.
\end{abstract}

\section{Introduction}

The 1990s upgrading of the Arecibo telescope and $2.38 \mathrm{MHz}$ radar system resulted in Arecibo having 20 to 30 times the sensitivity of the only other significant radar system used for solar system studies, the one on the $70 \mathrm{~m}$ NASA DSN Goldstone antenna [1] . Arecibo's sensitivity means that it is the dominant instrument for high quality Earth-based radar imaging and characterization of near-Earth objects (NEOs), imaging the terrestrial planets and studying the radio wavelength reflection properties of outer planet satellites and rings. Observations over the past decade have provided characterization and astrometric measurements of 20 to 25 near-Earth asteroids (NEAs) per year, opened up the field of NEAs as multiple body systems, verified the existence of the Yarkovsky effect and contributed to the verification of the YORP effect, obtained the first non-spacecraft images of cometary nuclei including an apparent contact binary, imaged a number of main belt asteroids (MBAs), studied the radar reflection properties of Titan and the mid-sized satellites and rings of Saturn, and obtained multi-polarization imagery of the Moon and terrestrial planets including high resolution imagery of the distribution of ice deposits at the poles of Mercury.

Radar imaging is the only ground-based technique capable of spatially resolving surface features of a large number of near-Earth objects, providing insight into the near-Earth asteroid (NEA) population. Radar imaging reveals the shape and spin state, which constrains the interior structure: rubble pile, coherent rock, thick regolith or none. This characterization is critical to hazard assessment and mitigation, necessary for understanding dynamical and collisional evolution in the solar system, and invaluable in planning for spacecraft missions. Additional solarsystem studies include observations of main belt asteroids, astrometry on a group of NEAs in highly eccentric orbits with small perihelion distances for tests of General Relativity, measurements of the Yarkovsky effect and measurements of solar oblateness, observations of cometary nuclei and their large particle comas. Studies of the terrestrial planets and the Moon continue with high spatial resolution, multi-polarization mapping of the near side of the Moon and similar measurements on Mercury, Venus and Mars, mapping polar ice deposits, looking for volcanic activity and probing surface and near-subsurface roughness, respectively.

\section{Near-Earth Asteroids}

For NEA observations, the Arecibo system can obtain delay-Doppler imagery with resolution as fine as $7.5 \mathrm{~m}$, a capability unmatched by any other technique short of a spacecraft mission (Fig. 1). These images can in most cases be used to determine directly the size, basic shape, and spin rate of an asteroid in a single day of observations. 


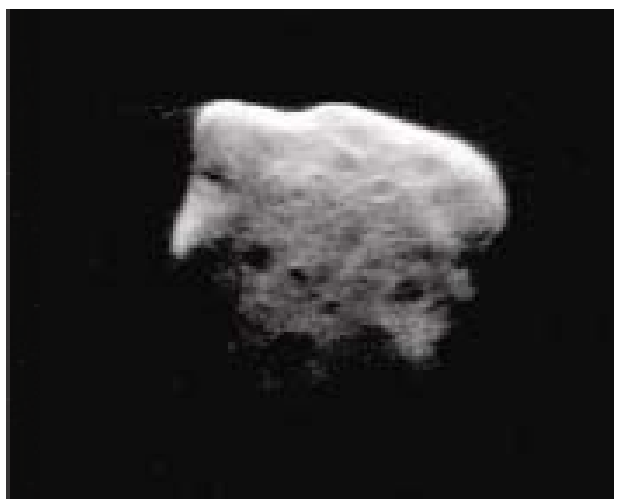

Fig. 1: Arecibo $15 \mathrm{~m}$ resolution delay-Doppler image of the $3.5 \mathrm{~km}$ NEA 1999 JM8 (Image courtesy of L. Benner, JPL)

A sequence of observations over several observing sessions can be used to obtain a very detailed shape model plus the pole direction via least squares fitting techniques (Fig. 2). The complementary astrometric data used for precise orbit determinations has accuracies of a few meters in range and millimeters per second in radial velocity. The Arecibo Observatory, with its great sensitivity and a well-established commitment to observing targets of opportunity whenever possible through the utilization of flexible scheduling techniques, has, over the last 8 years, typically observed $20-25$ NEAs per year with about half of those being "targets of opportunity" scheduled within days or hours of discovery.

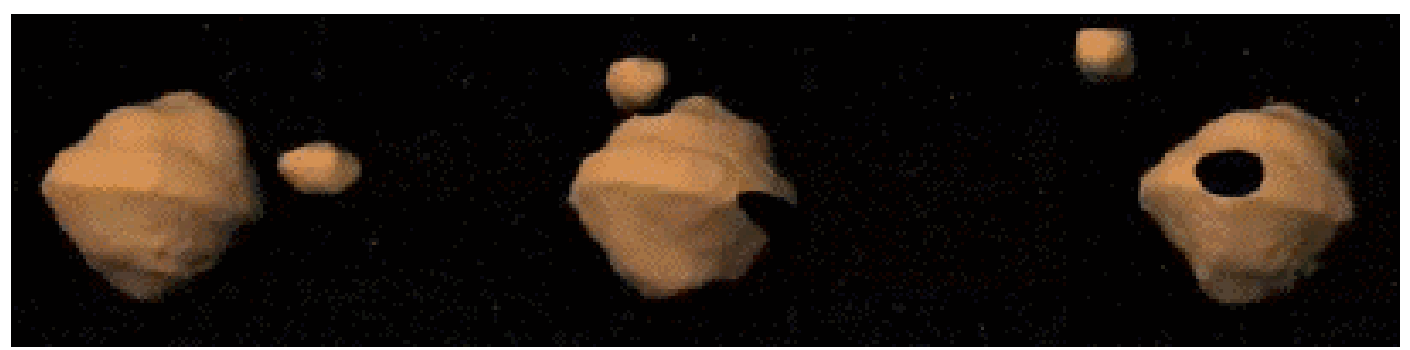

Fig. 2: $1999 \mathrm{KW}$, the first shape model of a binary near-Earth asteroid [2]. The black solar shadow is the result of an actual orbit simulation.

The rate of discoveries is expected to increase over the next few years through current programs such as the Catalina sky survey, results from WISE and the startup of Pan-STARRS over the next year and, eventually LSST and/or Pan-STARRS-IV. Such a leap in the known NEA population should be accompanied by a similar leap in our understanding of NEAs and solar system dynamics. There will be no shortage of potential targets, although the median size of new discoveries is likely to go down so that we may be working with many objects in the 100- to $300-\mathrm{m}$ size range. Radar observations will provide detailed shape and size information for the population, as well as precise astrometry to determine an unambiguous orbit solution providing many years of orbit prediction accuracy in a very short (few-day) interval. A single radar range and velocity measurement, when combined with plane-of-sky optical observations during a single apparition, will extend, on average, accurate orbit predictions by a factor of five, from $\sim 80$ to $\sim 400$ years, compared with just single apparition optical measurements [3]. This capability allows the probability of impacts associated with potentially hazardous asteroids (PHAs) to be quickly assessed and, hopefully, significantly reduced or eliminated.

The range of sizes and shapes is surprising, and the discovery of abundant (15\%) binary systems [4], triple asteroid systems [5], both ultra-fast and ultra-slow rotators with spin periods spanning minutes to weeks, and several (10\%) slowly-rotating candidate contact binaries [6] have revolutionized our understanding of these small bodies. Continued improvement in NEA discovery surveys, the advent of the Pan-STARRS program and results from WISE will extend the catalog of known objects. 
Torques applied by radiation forces at non-radial directions can either spin an object up or slow it down, depending on the geometry and obliquity [7]. Studies are underway to understand the details of forming binary asteroid systems by this process of spin-up, but in general it seems to explain the frequency, size ratios, and orbit characteristics that are observed. The effects of the YORP and Yarkovsky (a drift in semi-major axis due to reradiation of absorbed sunlight first validated by Arecibo and Goldstone radar astrometric observations [8]) processes have revolutionized our understanding of not only NEAs, but also main-belt asteroids and solar system formation. In addition to characterization for their own sake, NEAs can be used as "test particles" via precise astrometric measurements for studies of the forces on small bodies in the solar system, for tests of General Relativity and for measurements of solar oblateness.

\section{Comets}

Radar echoes from a comet nucleus can be used to directly measure (or constrain) nucleus size, shape, rotation, and surface density and measure the properties of the large $(>\mathrm{cm}$-sized) particle coma. Nine comets have been detected since 1998 with Arecibo. The three most recent of these detections produced nucleus delay-Doppler images, including images revealing Comet 8P/Tuttle to be a contact binary (Fig. 3; [9]) and 103P/Hartley 2 to be an elongated body, a result supported by the EPOXI flyby of this comet in November 2010. Other potential radar-

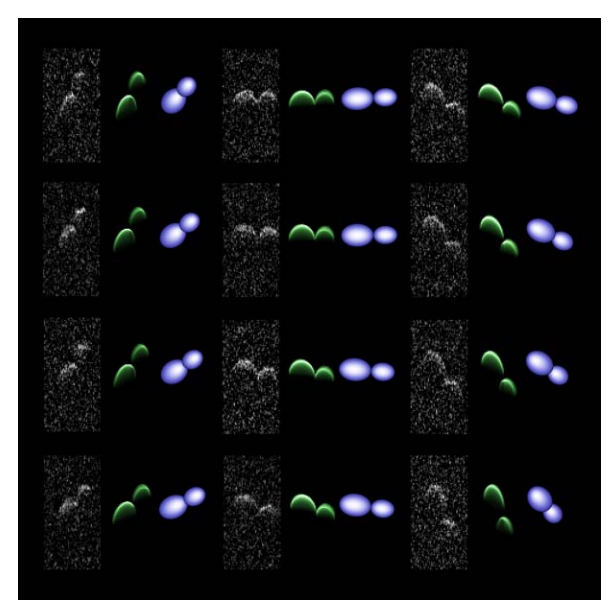

Fig. 3: Delay-Doppler images of Comet $8 \mathrm{P} /$ Tuttle at different orientations $(\mathrm{B} / \mathrm{W})$, the model of the system derived from the delay-Doppler images (blue) and the synthesized delay-Doppler images derived from the model (green) for comparison with the data. Image courtesy of John Harmon, NAIC.

detectable comets over the next five years include 45P/Honda-Mrkos-Pajdusakova ( $\Delta=0.25$ AU) in September 2011; P/2006 T1 (Levy) (0.19 AU) in January 2012; 189P/NEAT (0.19 AU) in July 2012; and 209P/LINEAR (0.06 AU) in May 2014. We hope to supplement these known opportunities with newly discovered objects, a likely prospect given that eight of the 13 comets detected by radar since 1980 were new.

\section{Other Programs}

Radar observations of main-belt asteroids (MBAs) concentrate on compositional tests based on bulk density and metal content and on shape determination [10]. Main-belt observing opportunities are rarer than NEO opportunities with imaging opportunities arise about twice per year. Particularly good imaging opportunities for 342 Bamberga in 2013 and 4 Vesta will occur in 2014.

Arecibo will continue to be used for studies of the likely ice deposits in shadowed craters at the poles of Mercury. Short of an orbiting radar system, the Arecibo's imaging capability will remain the only way to map these deposits [11]. Ongoing Mars observations are being used to image volcanic and other features at $3 \mathrm{~km}$ resolution.

Detecting active volcanism on Venus is a major objective of studies of this planet. Long-term monitoring with Arecibo has the possibility of detecting changes on the surface related to volcanic activity. The installation of 
an S-band receiving system on NRAO's upgraded Very Large Array (EVLA) opens the possibility of much higher quality imagery of Venus using the EVLA as a receiving antenna.

Arecibo is currently being used to obtain high resolution, multi-polarization imagery of the Moon using both the $13 \mathrm{~cm}$ (S-band) and $70 \mathrm{~cm}$ radar systems. This program will continue until the entire near-side of the Moon has been imaged.

\section{Acknowledgements}

The Arecibo Observatory is part of the National Astronomy and Ionosphere Center which is operated by Cornell University for the U. S. National Science Foundation and with support from the U. S. National Aeronautics and Space Administration.

\section{References}

1. S. J, Ostro, R. S. Hudson, L. A. M. Benner, J. D. Giorgini, C. Magri, J. L. Margot, and M. C. Nolan, "Asteroid Radar Astronomy," in Asteroids III, W. F. Bottke Jr., A. Cellino, P. Paolicchi, and R. P. Binzel (eds), Univ. of Arizona Press, Tucson, 2002, pp. 151-168.

2. S. J. Ostro, J. L. Margot, L. A. M. Benner, J. D. Giorgini, D. J. Scheeres, E. G. Fahnestock, S. B. Broschart, J. Bellerose, M. C. Nolan, M. Magri, P. Pravec, P. Scheirich, R. Rose, R. F. Jurgens, E. M. De Jong, and S. Suzuki, "Radar Imaging of Binary Near-Earth Asteroid (66391) 1999 KW4," Science, 314(5803), 2006, pp. 1276-1280.

3. S. J. Ostro and J. D. Giorgini, "The Role of Radar in Predicting and Preventing Asteroid and Comet Collisions with Earth," in Mitigation of Hazardous Comets and Asteroids, M. Belton, T. H. Morgan, N. Samarasinha, and D. K. Yeomans (eds), Cambridge University Press, 2004, pp. 38-65.

4. J. L. Margot, M. C. Nolan, L. A. M. Benner, S. J. Ostro, R. F. Jurgens, J. D. Giorgini, M. A. Slade, and D. B. Campbell, "Binary Asteroids in the Near-Earth Object Population," Science, 296(5572), 2002, pp. 1445-1448.

5. M. C. Nolan, E. S. Howell, T. M. Becker, C. Magri, J. D. Giorgini, and J. L. Margot, "Arecibo Radar Observations of 2001 SN263: A Near-Earth Triple Asteroid System," American Astronomical Society, DPS Meeting \#40, abstract \#25.04, BAAS, 40, 2008, p. 432.

6. L. A. M. Benner, M. C. Nolan, S. J. Ostro, J. D. Giorgini, D. P. Pray, A. W. Harris, C. Magri, and J. L. Margot, "Near-Earth Asteroid 2005 CR37: Radar Images and Photometry of a Candidate Contact Binary," Icarus, 182(2), 2006, pp. 474-481.

7. W. F. Bottke, D. Vokrouhlický, D. P. Rubincam, and D. Nesvorný, "The Yarkovsky and YORP Effects: Implications for Asteroids Dynamics," Ann. Rev. Earth and Plan. Sci., 34, 2006, pp. 157-191.

8. S. R. Chesley, S. J. Ostro, D. Vokrouhlický, A. A. Hine, L. A. M. Benner, and A. Chamberlin, "Direct Detection of the Yarkovsky Effect by Radar Ranging to Asteroid 6489 Golevka," Icarus, 302(5651), 2003, pp. 1739-1742.

9. J. K. Harmon, M. C. Nolan, and J. D. Giorgini, "Radar Observations of 8P/Tuttle: A Contact Binary Comet," Icarus, 207(1), 2010, pp. 499-502.

10. S. J. Ostro, C. Magri, L. A. M. Benner, J. D. Giorgini, M. C. Nolan, A. A. Hine, M. W. Busch, and J. L. Margot, "Radar Imaging of Asteroid 7 Iris," Icarus, 207(1), 2010, pp. 285-294.

11. J. K. Harmon, M. A. Slade, and M. S. Rice, "Radar Imagery of Mercury's Putative Polar Ice: 1999-2005 Arecibo Results," Icarus, 211, 2010, pp. 37-50. 